Kaygl, 20(I)/2021: 92-109. Araştırma Makalesi | Research Article

Makale Geliş | Received: 11.09.2020

Makale Kabul | Accepted: 22.11.2020

Yayın Tarihi | Publication Date: 15.03.2021

DOI: $10.20981 /$ kaygi.886200

\author{
Emre ŞAN \\ Doç. Dr. Assoc. Prof. Dr. \\ İstanbul 29 Mayıs Üniversitesi, Edebiyat Fakültesi, Felsefe Bölümü, İstanbul, TR \\ Istanbul 29 Mayis University, Faculty of Litterature, Department of Philosophy, Istanbul, TR \\ ORCID: 0000-0003-2654-9707 \\ esan@29mayis.edu.tr
}

\title{
Hareketin Fenomenolojisi ve Ontolojisi: Patočka'nın Aristoteles Okuması
}

Öz

Sorusu olmayan bir fenomenoloji düşünülemez ve fenomenolojik araştırmanın verimliliği ancak ortaya attığ1 sorularla ölçülebilir. Husserl ve Heidegger'in öğrencisi Jan Patočka’nın ilgilendiği temel soru bütünlük olarak dünya ve onu oluşturan hareketlerdir. Bu bağlamda yol gösterici argümanımızı şu şekilde ortaya koyabiliriz: Patočka, Aristoteles'in hareket teorisini radikalleştirerek, Husserl ve Heidegger'in ötesine uzanan bir ontolojik istikamette, dünyanın varlık anlamını kozmolojik tezahür hareketi olarak belirler. Şu halde öncelikle Patočka'nın, Husserl ve Heidegger'in arasında bir tür sentezi mümkün kılacak ve söz konusu Alman düşünürlerin felsefelerinde görünür olmayan ortak bir zemini açığa çıkaracak fenomenolojik felsefe projesini inceleyeceğiz. Ardından Aristoteles'in hareket teorisi üzerine Patočka'nın ortaya koyduğu yorumu ele alacağız. Söz konusu okuma Patočka'nın Husserl ve Heidegger'de üzeri örtülü bir biçimde devam ettiğini düşündüğü öznelcilik sorununun aşılmasında etkili olacak. Son olarak ise Patočka'nın Aristoteles'in hareket teorisini nasıl radikalleştirdiğini göreceğiz. Böylece tezahür hareketi olarak kozmolojik hareket teorisi açıklığa kavuşacak.

Anahtar Kelimeler: Fenomenoloji, Aristoteles, Hareket, Tekilleşme, Dünya.

\section{Phenomenology and Ontology of Movement: Patočka Reader of Aristotle}

Abstract

There is no phenomenology without problems and the fertility of phenomenological research can only be determined by the questions it raises. A disciple of Husserl and Heidegger, Jan Patočka is interested in the question of the world as a whole and the movements that compose it. The research hypothesis which guides us is then the following: we believe that by radicalizing Aristotle's theory of movement, Patočka thematizes the sense of being of the world as a cosmological movement of manifestation, following the path of an ontology beyond Husserl and Heidegger. We will first present the phenomenological philosophy of Patočka as a form of synthesis between Husserl and Heidegger. Then, we will focus on the Patočkian interpretation of movement in Aristotle. We will see how Patočka recaptures Aristotelian thought as a way to overcome the subjectivist impasse which is at work in Husserl and Heidegger. Finally, we will thematize the Patockian radicalization of Aristotle's theory of movement. This will allow us in particular to highlight a theory of cosmological movement as movement of manifestation.

Keywords: Phenomenology, Aristotle, Movement, Individuation, World. 


\section{Phénoménologie et ontologie du mouvement: Patočka lecteur d'Aristote}

\section{Résumé}

Il n'y a pas de phénoménologie sans problème, et la fécondité de la recherche phénoménologique ne peut etre jugée que par les questions qu'elle soulève. Disciple de Husserl et de Heidegger, Jan Patočka s'intéresse à la question du monde comme totalité et des mouvements qui le composent. L'hypothèse de recherche qui nous guide est alors la suivante: nous croyons qu'en radicalisant la théorie du mouvement d'Aristote, Patočka thématise le sens d'être du monde commme mouvement cosmologique de manifestation, selon la voie d'une ontologie se situant par-delà Husserl et Heidegger. Nous présenterons dans un premier moment, la philosophie phénoménologique de Patočka qui consiste à rendre possible une forme de synthèse entre Husserl et Heidegger et qui met au jour un terrain qui leur est commun, même s'il est demeuré caché à l'un et à l'autre. Puis, nous nous pencherons plutôt sur l'interprétation patočkienne du mouvement chez Aristote. Nous verrons, en quel sens Patočka ressaisit la pensée aristotélicienne comme une voie permettant de dépasser l'impasse subjectiviste qu'il croit encore à l'œuvre chez Husserl et Heidegger. Finalement, nous thématiserons la radicalisation patockienne de la théorie du mouvement d'Aristote. Ceci nous permettra notamment de mettre en évidence une théorie du mouvement cosmologique comme mouvement de manifestation.

Mots-clès: Phénoménologie, Aristote, Mouvement, Individuation, Monde.

\section{Philosophie phénoménologique}

Dans ses travaux tardifs sur Platon et l'Europe, Patočka effectue une distinction claire entre la phénoménologie et la philosophie phénoménologique. D’après lui, «la phénoménologie est la science des phénomènes en tant que telle, elle ne nous montre pas les choses mais le mode de donation des choses »(Patocka 1997: 39). Une philosophie phénoménologique s'intéresse, quant à elle, à la légalité et à la structure de l'apparaître qui sont indépendantes de la structure des choses, et au fond, c'est seulement à ce niveau que la phénoménologie se justifie comme une discipline autonome. En ce sens, la loi de l'apparaître n'a rien à voir avec la loi de l'apparaissant : les lois qui règnent au sein des choses manifestées ne sont pas les lois de leur manifestation. Cela implique que la phénoménologie doit être conçue hors de toute forme de réalisme naturaliste et de psychologisme, parce que le naturalisme rend compte de la structure de l'apparaître à partir des lois de la nature, et que le psychologisme rend compte des lois de l'apparaître à partir des lois de la psychè, de la conscience empirique. La phénoménologie suppose une critique radicale du naturalisme et du psychologisme, dans la mesure où, pour elle, la légalité de l'apparaître est 
indépendante de toute légalité naturelle quelle qu'elle soit. Comme l'écrit Patočka de manière aussi précise que synthétique :

[L]a philosophie phénoménologique se distingue de la phénoménologie dans la mesure où elle ne veut pas seulement analyser les phénomènes en tant que tels, mais encore en tirer des conséquences «métaphysiques » et pose la question du rapport entre le phénomène et l'étant, les étants [...]. Il y a des conceptions qui estiment que le phénomène est en son fond une subjectivité sui generis, de la vie, du vécu. À mon avis, c'est mécomprendre, passer à côté du problème propre du phénomène en tant que tel. Le phénomène doit demeurer phénomène, il doit demeurer un domaine autonome, non-réel de l'univers, un domaine qui, bien qu'il ne soit pas réel, détermine d'une certaine manière la réalité (Patocka 1997 : 41).

Une telle entreprise depasse donc le plan phénoménal pour interroger la nature des étants et pour analyser l'étant à la seule lumière de sa phénoménalité. Toutefois la phénoménalité est constitutive de l'être réel sans que pour autant l'étant s'épuise dans sa phénoménalité. Il s'agit d'un domaine non-réel car il s'oppose à la réalité comprise comme ce qui n'existe que comme pleinement déterminé. Autant dire que si la phénoménalité est distincte de l'être réel, elle est d'une certaine façon nécessaire à l'être réel, car l'être réel ne pourrait pas se phénoménaliser s'il restait totalement étranger à la phénoménalité. Une phénoménologie qui distingue la sphère de l'apparaître de la sphère de l'apparaissant est donc naturellement conduite à une interrogation métaphysique qui caractérisera la structure de l'étant réel en tant que codéterminé par sa manifestation, et ce dans la mesure où cette manifestation sera un mode d'accomplissement de l'être.

Patočka rejoint ici Heidegger dans sa théorie de la différence ontologique. Il écrit : « la manifestation se produit dans le domaine de l'être, de quelque chose qui $n$ 'est pas un étant, dans un domaine essentiellement non-étant. Les choses ne peuvent se montrer que sur ce fondement»(Patocka 1997: 180). C'est ainsi que la phénoménologie devient phénoménologie ontologique, phénoménologie de la différence ontologique. Selon Heidegger, la chose même à quoi la phénoménologie fait revenir la philosophie ne se nomme plus l'étant (ni l'essence, ni la catégorie, ni le noème, etc.) mais, plus 
radicalement, l'étant en vue de l'être. Cependant, Patočka n'en reste pas là et il se situe par-delà l'approche heideggérienne. Selon lui en effet:

[L]e problème de la manifestation est plus profond, plus fondamental, plus originel que le problème de l'être. Tout simplement parce que je ne peux venir au problème de l'être qu'à travers le problème de la manifestation, au lieu que si je pars du problème de l'être au sens abstrait du terme, le concept d'être devient pour moi un concept abstrait quelque chose comme un signe purement formel ; pas même une catégorie, mais quelque chose qui vient au-dessus des catégories en ce sens qu'il est entièrement dépourvu de contenu (Patocka 1997 : 177).

Autrement dit, si la question de l'être doit effectivement être posée, elle est néanmoins seconde par rapport à la question de la manifestation. Patočka souligne ainsi sa volonté de proposer une nouvelle interprétation de la manifestation: l'être de l'étant doit être abordé par-delà sa phénoménalité (contre Husserl), mais aussi comme cela qui est co-déterminé par la phénoménalité (contre Heidegger) ${ }^{1}$ :

Pour Husserl, l'étant vrai n'est pas autre chose que le phénomène, tandis que pour Heidegger le phénomène est phénomène de l'étant, manifestation d'un étant réel dont la compréhension, la clef, est à chercher dans le phénomène profond. Cette différence fondamentale est la raison pour laquelle chez Heidegger il ne s'agit pas d'un ressouvenir mais de l'apparition au sens fort du terme (Patocka 1997 : 183).

Patočka se situe ainsi à égale distance de la phénoménologie de Husserl, qui emprunte les lois de l'apparaissant pour rendre compte des lois de l'apparaître, et de la phénoménologie ontologique de Heidegger, qui ne peut se désolidariser de la phénoménalité du plan de l'étant qu'en la référant à l'être. En revanche, dans la perspective de Patočka, l'apparaître échappe à l'étant et à l'être: la structure d'apparaître ne se confond pas avec la structure d'un apparaissant, mais sans être pour autant reconductible à l'être. En d'autres termes, le problème de l'apparaissant et le

\footnotetext{
${ }^{1}$ En fait, dans les manuscrits qui portent le titre «Epochè et Réduction», (Patočka 1995 : 163-21), Patočka explicite le motif qui le distingue de Heidegger et le rapproche et oriente vers une radicalisation de Husserl. Comme il le souligne, «[P]our Heidegger, la phénoménologie n'est pas une théorie de l'apparaître en tant que tel, mais des phénomènes. Phénomène tout d'abord formel : ce qui se montre de soi-même. Le phénomène est toujours orienté vers l'étant et ce qui en soi lui appartient » (Patočka 1995 : 207).
} 
problème de l'être sont des problèmes prématurés, qu'il faudrait repenser une fois posée la question première de l'apparaître.

C'est dans l'horizon propre à la «philosophie phénoménologique » du problème de l'apparaître que Patočka interprète la détermination heideggérienne de l'être de l'étant. Pour ce faire, il s'intéresse de près à ce qu'a découvert le «regard phénoménologique » de Heidegger dans sa lecture d'Aristote. Selon lui, l'innovation de Heidegger consiste à montrer que, déjà chez les penseurs de l'Antiquité, tel Aristote, l'être n'est pas conçu comme un moment de l'étant, mais qu'il s'agit en réalité du mode d'apparition des choses. Comme l'affirme Pierre Rodrigo:

[C]ette intuition de l'expérience grecque de l'alètheia conduisit directement Heidegger à interroger le sens de l'être. Si, en effet, le «se-montrer» de l'étant peut se dire en grec «non-retrait», c'est que la présence constante s'est d'emblée imposée comme sens déterminant de l'être de l'étant qui, en vérité, est (Rodrigo 1995 : 168).

Une telle intuition est confirmée par l'analyse du sens de l'ousia. Le questionnement sur la forme caractéristique de la chose nous invite en effet à penser que l'ousia, ce que l'on appelle la substance, se détermine en ce qu'elle a une limite, en ce qu'elle se délimite d'une certaine manière, et que cette délimitation, cette entrée dans une limite signifie que c'est la chose même qui se définit. Cette délimitation du caractère de la chose elle-même correspond à son découvrement. Patočka, via Heidegger, attribue à Aristote le mérite fondamental d'avoir pensé l'être comme identique au se montrer comme délimitation :

La non-occultation n'advient pas à l'étant, aux choses étantes, grâce à notre seul jugement. Au contraire, tout énoncé sur l'étant n'est possible que si celui-ci est en quelque sorte d'avance dévoilé, s'il s'est déjà manifesté. La non-occultation, la manifestation, la manifesteté, est donc un trait de l'étant lui-même. Aussi Aristote peut-il dire que l'étant et le non-voilé sont identiques. Ce n'est pas une subjectivation de l'étant, cela ne veut pas dire que l'étant soit créé par nous. Même la délimitation, le dévoilement, n'est pas le résultat de notre acte ou jugement, mais quelque chose qui se produit dans les choses mêmes (Patocka 1997 : 170). 
Comprenons bien ces lignes : la manifestation comme délimitation procède de l'étant lui-même et non pas d'un sujet; autrement dit, la subjectivité n'est pas la condition de possibilité de la délimitation, mais ce qui est rendu possible par la délimitation $^{2}$. Ainsi philosophie phénoménologique de Patočka, dépasse la caractérisation statique de l'être, puisqu'il définit l'être comme apparaître et l'apparaître comme un mouvement phénoménalisant qui a pour fonction l'«entrée dans l'apparition » (Patocka 1995 : 127), autrement dit, l'individuation. Pour comprendre la manifestation comme individuation, nous devons éviter toute référence à un principe positif pour penser l'individualité et renoncer aux deux approches classiques de l'individuation. D'une part, l'approche substantialiste qui consiste à rendre compte de l'être comme consistant en son unité, donné à lui-même, fondé sur lui-même et résistant à ce qui n'est pas lui-même. D'autre part, l'approche hylémorphique qui consiste à rendre compte de l'individu comme engendré par la rencontre d'une forme et d'une matière. Contrairement aux deux approches classiques de l'individuation, Patočka propose une troisième voie et explique qu'une manifestation advient toujours à partir d'un sens dynamique de l'individuation. En ce sens, en tant que « facteur ontologique fondamental » (Patočka 1988: 129), le mouvement est le fondement de toute manifestation. En référant la manifestation à un mouvement effectif procédant de l'être lui-même, on écarte le risque de référer la manifestation à un sujet ou à un étant qui porterait la charge de la phénoménalité. Ainsi, la seule manière de surmonter le transcendantalisme subjectiviste fondé sur une conception préalable du sujet, de la réflexion et du temps, est de fonder l'activité du sujet sur une manifestation préalable et de comprendre la manifestation comme individuation. Ce mouvement fondamental de l'apparaître implique à la fois un mouvement primaire (ou «proto-mouvement ») et un

\footnotetext{
${ }^{2}$ Patočka renverse ici toute une tradition selon laquelle le phénomène est interprété comme représentation (l'étantité renvoie à l'objectité qui renvoie à une représentation, à une subjectivité). Car, comme il le souligne, « au point de vue de la phénoménologie, le concept de "représentation" ne correspond à rien du tout; le représenter est la possibilité subjective d'avoir une apparition non originaire de l'étant, et le percevoir la présence de l'objet en original. Le terme "représentation" relève du vocabulaire, non pas d'une réflexion sur les phénomènes, mais d'une manière de voir constructive » (Patočka 1995: 119). Dans la perspective de Patočka, l'être conçu de l'étant renvoie ainsi à son être objet, et son être objet repose sur la délimitation (donc la subjectivité renvoie à l'objectité qui renvoie à l'étantité).
} 
mouvement subjectif, en quelque sorte secondaire. Autrement dit, l'ouverture originaire dans et par laquelle les étants adviennent, ouverture individuante ou séparante, doit ellemême être comprise comme mouvement. Il y a un mouvement de la manifestation qui individualise ou délimite l'étant, le conduit à lui-même en le séparant du fond dont il provient, et ce mouvement est l'advenir même de l'étant en son étantité. Il faut admettre, ensuite, que le mouvement subjectif prolonge nécessairement ce mouvement originel : le mouvement de l'existence comme mouvement de phénoménalisation de l'étant renvoie au mouvement même du monde comme mouvement de surgissement de l'étant. La manifestation au sujet procède donc d'une première manifestation « en soi », d'une entrée dans la singularité, mais c'est le même «mouvement » qui s'accomplit à ces deux niveaux de la manifestation. Comme le souligne Patočka :

L'apparaitre comme sortie hors du fondement obscur: qu'il y a ici un mouvement de l'apparaître, un proto-mouvement, c'est ce qu'atteste per analogiam l'étude de l'apparition secondaire, de l'apparition de l'apparaissant qui présuppose une création de centres, la constitution d'une centralité : le mouvement de transcendance crée ici un «monde propre », monde ambiant... De même, il doit y avoir quelque chose comme un mouvement par lequel le cœur du monde constitue son contenu contingent et dont l'espace-temps-qualité en totalité est un sédiment (Patocka 1995 : 157).

Autrement dit, il y a nécessairement un proto-mouvement qui, surgissant du monde et le déployant en ses moments individués, donne lieu à un second mouvement qui fait surgir le monde comme monde paraissant à... Ce double mouvement phénoménalisant représente les deux dimensions d'un seul et même mouvement, qui se rapportent toutes deux au monde mais sur un mode différent. Le mouvement primaire ou «proto-mouvement» consiste en un mouvement d'individuation et il correspond à une désubjectivation de la manifestation.

\section{L'appropriation patočkienne d'Aristote : la priorité du mouvement}

Les investigations ontologiques de Patočka sur le mouvement sont guidées par l'ambition aristotélicienne de trouver une commune mesure entre le monde et l'homme. Le mouvement en vient à fournir une détermination ontologique fondamentale: 
irréductible à un quelconque sens intramondain et ontique, le mouvement est conçu comme procès onto-génétique, c'est-à-dire comme advenue à l'apparaître. Selon Patočka, le plus grand apport de la Physique tient à l'établissement des conditions d'un tel mouvement, qui a pour déploiement vivant la nature (phusis) et pour fond le monde (kosmos). Il nous faut nous pencher à présent sur la façon dont Patočka comprend et actualise certains problèmes aristotéliciens, en les repensant en relation avec les questions fondamentales qu'il affronte dans son propre horizon phénoménologique.

La notion de phusis va acquérir dans la Grèce classique, avec Aristote, sa forme rationnelle la plus achevée. La conception aristotélicienne gardera son hégémonie jusqu'à l'arrivée, au XVII ${ }^{\text {ème }}$ siècle, de la philosophie et de la physique modernes, même si, de par certains de ses postulats épistémologiques les plus profonds, elle continuera à occuper une place privilégiée jusqu'à nos jours. Chez Aristote, l'étude des êtres naturels se définira comme l'étude des êtres qui ont en eux la cause de leur mouvement. Ce type d'êtres est celui des êtres vivants - les hommes, les plantes et les animaux - mais également celui des corps terrestres composés des quatre éléments. C'est pourquoi l'étude des choses qui sont par nature s'étendra à tout ce qui est soumis au mouvement, et inclura des disciplines comme la zoologie et l'optique, la biologie et la météorologie.

Commençons par préciser la teneur de la question du mouvement: comment peuton penser le mouvement? On définit le plus souvent la pensée comme ayant pour but de définir l'essence, l'invariant d'une réalité - c'est-à-dire ce qui ne change pas avec le temps et le lieu; dès lors, comment la pensée pourrait-elle s'appliquer au mouvement, puisque sa fin et sa méthode consistent à éliminer tout ce qui relève du changement? Elle ne semble pouvoir saisir le mouvement qu'à condition de déterminer ce qui, dans le mouvement, ne change pas et n'est donc pas en mouvement. Penser le mouvement reviendrait ainsi à éviter soigneusement de penser ce qui, dans le mouvement, est vraiment mouvement. La critique parménidienne du mouvement vient précisément d'une telle séparation entre pensée et mouvement, corrélative d'une identification de la pensée, de l'être, et du même: l'être est ce qui a une stabilité essentielle, et l'acte de penser, précisément, porte sur et vient de l'être, tandis que le mouvement n'est rien. Être 
et penser sont par suite le même; ce qui est pensable est, ce qui est impensable n'est pas $^{3}$. En fait, si comme l'affirme Parménide, seul ce qui est peut être pensé, il y a dans le concept de non-être quelque chose de contradictoire et d'impensable. Or, tout mouvement enveloppe un passage du non-être à l'être ou de l'être au non-être (que ce soit dans le sens absolu de la génération et de la corruption, ou dans le sens non absolu du déplacement), le mouvement devient donc impensable, et partant, non-être. La physique moderne ${ }^{4}$ a toujours cherché à mesurer le mouvement, mais jamais à le définir; à cet égard, la perspective d'Aristote est confrontée à un obstacle: le mouvement paraît impossible à définir puisque la pensée définit l'essence éternelle, immobile et que le mouvement est la négation même de l'éternel et de l'immobile. Mais, Aristote tente précisément de répondre à Parménide: il cherche à montrer que le mouvement est une réalité dotée d'une certaine consistance, qui peut donc donner lieu à un savoir. C'est la possibilité de fonder une «science de la nature » qui est ici en jeu, la science de la nature étant science des étants qui sont en mouvement et en repos. La démarche

\footnotetext{
${ }^{3}$ Zénon montre que toute tentative pour penser le mouvement aboutit à un paradoxe insurmontable, comme le paradoxe de la flèche: si l'on décompose le mouvement en instants successifs, en une série de points fixes - fixité inhérente à l'acte de penser, puisque penser, c'est décomposer - alors, on aboutit à une immobilité de la flèche à chaque instant, immobilité qui contredit son mouvement général. La signification profonde de la thèse de Zénon est que le mouvement n'est pas pensable et n'a pas d'être. Parménide établit la thèse fondamentale (être et penser sont le même) alors que son disciple Zénon oppose la contre-épreuve de la thèse: si l'on donne de l'être à ce qui n'en a pas, on en arrive à des paradoxes insurmontables. Autrement dit, la pensée ne pense que le fixe, ne peut penser le mouvement, de par sa nature même; la pensée serait donc décomposition, séparation des moments qui figerait le mouvement; mais est il possible de penser autrement la pensée - de penser une pensée non confinée au fixe ; et ne peut-on penser le mouvement de sorte qu'il ne soit pas opposé de manière radicale au fixe ? Il est un fait que le mouvement tel que le pense Parménide est pur mouvement, sans rien de fixe: s'il n'est en rien, c'est que nécessairement rien ne s'y conserve, on ne peut rien en saisir de fixe.Les Éléates semblent ici donner de manière anticipée une définition platonicienne du mouvement, mouvement qui ne serait rien que mouvement, pur mouvement, sans être. En effet, l'idée platonicienne de l'être est celle d'un être qui n'est que ce qu'il est, à l'exclusion de tout ce qu'il n'est pas; le mouvement devrait donc être pur mouvement, mouvement pur, qui ne serait que mouvement. C'est là une pensée abstraite du mouvement, qui ne permet pas de saisir les mouvements existants, réels, déterminés, observables dans la nature: un tel mouvement qui serait «pur» mouvement n'est-il pas une création, une abstraction pure qui d'ailleurs ne bouge pas ? Ce mouvement pur n'est jamais «vu à l'œuvre » dans la réalité. La pensée s'oppose donc chez Parménide, Zénon et Platon, non pas tant au mouvement qu'à la pensée $d u$ mouvement.

${ }^{4}$ Comme le souligne Patočka, «il existe une conception moderne du mouvement comme structure de paramètres quantitatifs continus et corrélatifs, paramètres du trajet, du temps, de la vitesse, de l'accélération, etc. Cette conception procède d'une idéalisation objectivante du mouvement originel; elle ne contient rien du mouvement au sens propre; sa spécificité, en tant que structure quantitative, réside dans la possibilité qu'elle donne de prévoir et maîtriser le mouvement réel », (Patočka 1988 : 102 ).
} 
d'Aristote consiste à montrer qu'on peut effectivement élaborer une science de la nature, une science de ce qui est en mouvement. Aristote doit donc montrer que le mouvement est «pensable » et non pas simplement constatable. Le mouvement, dit-il, est «l'entéléchie de ce qui est en puissance, en tant que cela est en puissance» (Aristote, Physique, III, 1, 201 a 10s).

Chez Patočka, la reference à Aristote se justifie à deux niveaux : tout d'abord, c'est précisément à la signification ontologique inscrite dans ce passage de la puissance à l'acte que Patočka accorde une importance particulière : ce passage est un mouvement ontologique au sens où il est passage de la privation à la possession d'une détermination, où il est ce qui relie le déterminé et ses déterminations, à savoir ce qui amène la présence de la détermination dans le déterminé. De plus, en tant que réalisation d'une déterminité plus grande, il doit être compris, en son essence, comme produisant un accomplissement d'être : le mouvement est « la manière dont un être fini, qui ne peut exister pleinement, peut néanmoins connaître une existence maximale » (Patočka 2011: 252). Chaque être naturel n'arrivera ainsi au maximum de sa détermination - et ainsi au maximum d'existence qu'il doit atteindre - qu'à l'issue d'une série de mouvements qui le définiront en tant qu' «être-ceci » ou qu' « être-cela ». Le mouvement n'est, par suite, qu'un «passage de détermination en détermination» (Patočka 2011: 252) et, en ce sens, l'être naturel ne devient un être particulier, individuel que par sa capacité de mouvement.

Selon Patočka, il n'est pas question d'affirmer, comme l'on le fait le plus souvent, que la philosophie aristotelicienne de la nature, dominée par le schema téléologique de l'actualisation d'une essence, de l'accomplissement, releverait d'une simple projection anthropomorphique. Il interprete la conception aristotelicienne du mouvement dans un tout autre sens, convergent avec sa propre conception de la phénoménalité. Il ne s'agit donc pas de partir de la subjectivité et de sa différence vis à vis du monde pour ne voir dans la conception aritotelicienne qu'une projection de son mode d'être au sein des choses; il s'agit tout au contraire de ressaisir l'être du sujet à partir d'une modalité d'être plus générale, convenant à la totalité de l'étant, et dont la subjectivité n'est alors 
qu'une spécification: c'est bien l'homme lui-même qui doit être compris à la lumiere des structures de l'être.

Pour répondre à ce souci, Patočka reprend un des concepts qui, chez Aristote, avait ainsi vocation à jeter un pont entre l'humain et l'extra-humain, et fait le pari que c'est le mouvement qui constitue ce point de passage et permet donc de passer d'ontologies régionales à une véritable ontologie générale. Il s'agit dès lors de décrire l'être du mouvement sans le réduire, et toute la difficulté tient dans cette tentative de circonscrire l'être du mouvement tout en faisant droit à son extraordinaire diversité. Patočka met ainsi en place dans le texte une variation autour du concept du mouvement, hésitant entre variation eidétique et variation d'exemples, grâce à laquelle il passe en revue les différentes acceptions possibles du mouvement et tente de le distinguer des concepts proches tout en faisant droit à ses diverses manifestations. Une des principales difficultés est alors de faire face à l'éclatement apparemment irréductible des différents mouvements particuliers. Y a-t-il une quelconque communauté ontologique entre la chute d'un corps, un vol d'oiseau, une flèche lancée, la croissance d'une plante, la rotation de la terre ? C'est en effet seulement en comprenant le mouvement dans sa fonction individuante qu'il est possible de rapporter la diversité de ses manifestations à une unité d'essence. Le mouvement est lié au phénomène de l'individuation de l'être par lui-même, et c'est seulement ainsi qu'il peut se trouver en mesure de jouer le rôle unificateur, qu'on trouve déjà chez Aristote, de trait d'union ontologique entre l'homme et le monde. Le mouvement ne peut alors être compris que dans ce cadre, comme cela qui conduit la substance individuelle à elle-même et, par voie de conséquence, l'actualise aussi selon ces autres catégories que sont la quantité, la qualité et le lieu. Il faut enfin rappeler que, si la substance peut-être entendue en trois sens, c'est bien la quiddité, c'est-à-dire au fond la determination (ce qu'est l'étant), qui est la plus originaire. En tant qu'il affecte donc la substantialité de la substance, le mouvement consiste necessairement dans un procès de détermination: il est ce qui conduit la substance à sa quiddité. Autrement dit, le mouvement est ce qui occasionne les déterminations, ce qui produit leur être-ensemble, c'est-à-dire ce qui réalise une 
synthèse. Il n'est donc pas tant ce qui «produit » les déterminations que ce qui les unifie, cette unité réalisée qualifiant l'étant dans sa singularité. Dans cette optique, il n'y a pas de substrat déjà déterminé qui possèderait en puissance les déterminations, car un étant ne précède pas son mouvement. Le substrat ne devient substrat qu'en tant que ses déterminations se rassemblent en lui, c'est-à-dire qu'en s'accomplissant: le mouvement constitue donc le substrat lui-même. C'est pourquoi, Patočka ne suit pas le raisonnement d'Aristote, mais le radicalise.

\section{La radicalisation patočkienne d'Aristote : la priorité du mouvement sur le substrat}

En quoi consiste la radicalisation patockienne de la théorie du mouvement d'Aristote? Selon lui, « [1]'importance accordée par la conception aristotélicienne à la réalisation de la dynamis met en relief l'aspect protensif, l'avenir. Toutefois, Aristote localise la puissance dans un substrat censé rendre le changement possible en subsistant sans changement, en étant conservé à l'intérieur du changement. De ce fait, le mouvement est réintégré de force dans le simple présent, dans le temps comme succession d'instants formant un continuum linéaire » (Patočka 1988: 103). En réalité, pour que le mouvement soit protensif, c'est-à-dire temporalisé par le futur, et pour qu'il soit vraiment créateur, il est nécessaire qu'il ne reçoive pas son unité d'un substrat, et qu'il crée et déploie sa propre unité. C'est pourquoi Patočka poursuit :

Seule pourrait faire avancer la question une radicalisation de la conception aristotélicienne - le mouvement comme vie originelle qui ne reçoit pas son unité du substrat conservé, mais crée elle-même sa propre unité et celle de la chose en mouvement. Seul le mouvement conçu de cette manière est mouvement originel (Patočka 1988: 103).

Ce qui est essentiel est que, si le mouvement est le facteur ontologique fondamental et si le mouvement est le fondement de toute manifestation (comme individuation et dévoilement), alors il faut que l'unité des choses en mouvement soit réalisée ou créée par le mouvement lui-même, et non garantie par une unité substantielle préalable (il n'y a de substrat qu'en raison d'une unification préalable). Dans sa lecture 
d'Aristote, Patočka insiste donc sur le fait que le substrat ne précède pas mais, à l'inverse, requiert le mouvement. Dans cette perspective, le mouvement est la condition de l'identification d'un substrat: c'est par le mouvement qu'un individu se sépare, prend possession de lui-même et se distingue comme individuel. « Le mouvement est donc ce qui rend l'étant ce qu'il est. C'est le mouvement qui unifie, entretient la cohésion, synthétise les déterminations de l'étant » (Patočka 1995: 31). Dès lors, dans la mesure où l'être n'est rien d'autre que ses déterminations, le mouvement fait être l'étant en mouvement en lui apportant ses déterminations; il est ce qui permet à l'étant d'être ce qu'il est, il est mouvement ontologique. Autrement dit, le mouvement constitue l'étant dans son étantité, dans son ipséité, donc son individualité. Il est ce qui réalise le passage de l'être à l'étant. Il s'agit ici pour Patočka de transcender les catégories aristotéliciennes (de substance, de quantité, de qualité et de lieu) en faisant du mouvement substantiel l'essence de tout mouvement. Le mouvement de la substance (genesis-phthora) doit être conçu comme délivrant l'essence même du mouvement : Il s'agit par conséquent de comprendre le couple genesis-phthora, comme le modèle de l'essence de tout mouvement: tout mouvement doit être conçu comme un mode d'avènement d'un étant, comme un mode de réalisation d'un étant; tout mouvement est de l'ordre du mouvement substantiel, il y a donc un mouvement par lequel l'être advient, un mouvement qui fait qu'il y a quelque chose. Au final, il ne s'agit pas d'un mouvement dans l'être (qui serait divisé selon les catégories d'Aristote, mais il s'agit d'un mouvement de l'être et avec l'être, qui produit les catégories d'Aristote.

Cependant un pur accomplissement, tel que rien en lui ne demeureait le même, bref un accomplissement sans sujet, ne serait plus mouvement mais évènement: quelque chose arrive, par exemple « il pleut », mais ce n'est pas un mouvement, car cela n'arrive à personne, il n'y a aucun sujet qui s'accomplit en cet évènement. En ce sens, il n'y a pas de mouvement sans sujet et le mouvement doit par conséquent être defini comme cela qui est sans substrat, mais non pas sans sujet. Mais comment penser un procès qui est sans substrat, pour autant qu'il est de part en part changement, mais qui n'est pourtant pas sans sujet, sans quoi rien ne changerait, rien ne serait affecté par ce procès, 
si bien que nous basculerions alors du côte de l'évènement? La mobilité exige un sujet qui n'est pas immuable comme un substrat, mais qui est néanmoins constitué en son unité par le mouvement lui-même. Le geste proprement patočkien consiste alors à penser le mouvement non comme actualisation d'une puissance, mais comme réalisation d'une possibilité.

La manifestation n'est plus comprise comme la création paradoxale d'un déjà làd'un substrat qui est déjà en puissance les déterminations qui lui adviendront - mais comme la création d'une nouveauté. En revanche, cette nouveauté n'est jamais véritablement radicale puisqu'il ne peut y avoir de création ex nihilo par le mouvement: en tant qu'elle s'enracine nécessairement dans une possibilité, elle fait naître la possibilité en la réalisant. L'exigence d'une désubstantialisation de la puissance vient ainsi de la nécessité d'une inscription de l'étant dans l'horizon du monde qui le précède. Comme l'écrit Patočka, «[L]e mouvement est ce qui fait apparaître qu'il y a, pour un temps déterminé, une place dans le monde pour une réalité singulière déterminée parmi d'autres réalités singulières »(Patočka 1988: 103). La nouveauté introduite par Patočka réside en ceci que, chez lui, la théorie du mouvement ontogénétique comme constitution de l'étant s'articule profondément avec la manifestation. C'est précisément cette articulation qui donne la clé de cette affirmation énigmatique de Patočka. Au fond, il n’y a de détermination que dans et par le mouvement, lequel est « le fondement de toute manifestation ». Enfin, dire que le mouvement, en tant qu'il détermine, constitue l'étant tel qu'il est, revient à faire apparaître et à délimiter une place dans le monde pour cet étant, donc, le mouvement est essentiellement mouvement de manifestation. Plus précisément, le mouvement qui rend manifeste l'étant est au fond un mouvement qui fait apparaître une place, de sorte que faire apparaître un étant veut dire faire paraître sa place. Le mouvement distingue ou sépare, et ce faisant, il fait place à l'étant.

La manifestation n'est donc pas une production, une création, mais l'étant préexiste toujours à sa manifestation au sens où il est toujours déjà compris dans le fond du monde dont procède la manifestation, de sorte que manifester ne consiste qu'à «donner une place », c'est-à-dire à créer un lieu et non pas un étant. Penser le 
mouvement signifie aussi penser le caractère originaire de l'espace et, comme l'écrit le philosophe tchèque, «[1]e processus n'est pas nécessairement quelque chose de purement temporel. Le mouvement comme tel n'est jamais sans référence à l'espace » (Patočka 1995: 33). Autrement dit, l'effort patočkien pour éliminer l'antériorité ontologique du substrat dans le mouvement est lié au caractère originaire de la dimension spatiale: la manifestation n'a pas un sens positif de production mais un sens négatif qui consiste à dégager les obstacles pour faire place. En d'autres termes, la caractérisation de la manifestation comme délimitation fait écho à une position préspatiale et il s'agit, non pas de dévoiler, mais de constituer une place, de constituer le là et non pas de voir un déjà là. Il s'agit d'un sens négatif car la place est un vide d'étant, il convient de dégager les obstacles pour que l'étant puisse accéder à son là.

Finalement, ce premier geste va de pair avec un second aspect de la radicalisation. Patočka montre que chez Aristote le mouvement est toujours un mouvement depuis un « où » vers un autre « où » (Patočka 1988: 128), c'est-à-dire qu'il est une unité indivisible délimitée par le point d'arrivée et par le point de départ. L'unité n'est donc pas une unité substantielle, mais une unité donnée par la finalité du mouvement: cette finalité est ce pour quoi il y a un substrat. Le mouvement vise une finalité (un telos) qui dépasse celle de l'étant individuel. D'après cette vision « téléologique » de la réalité, le monde lui-même comme auto-manifestation serait la finalité du mouvement au sens cosmologique (c'est-à-dire au sens de la réalisation finale à laquelle se subordonnent toutes les autres). Cependant, le caractère téléologique du mouvement pose un problème capital. En effet, dans la mesure où le mouvement rassemble les différentes déterminations d'un déterminé en vue d'obtenir leur actualisation maximale, il est le maximum de présence possible en tant que tel qui est visé. Néanmoins, cette présence de l'étant n'a pas un caractère exclusivement positif et l'aboutissement de la forme dans sa détermination maximale demeure toujours approximatif. En fait, le processus de déterminabilité qu'effectue l'étant, lorsque se réalisent en lui ses déterminations potentielles, exige une cause qui active ce passage, qui mette en mouvement le mouvement. Cependant, la forme est identique à la quiddité (to ti en einai), c'est-à-dire 
à ce par quoi une chose se définit; mais elle coïncide également avec la fin (telos), qui n'est autre que la forme déployée et que l'être en question doit atteindre pour être luimême (entelecheia). À cet égard, l'eidos est en même temps le principe d'organisation d'une chose, l'idéal auquel tend cette chose lors de son actualisation, et la force même qui met en mouvement le processus qui lui permet de devenir ce qu'elle est. La forme, considérée de manière dynamique comme energeia, n'est donc pas seulement un état de pleine actualisation, mais encore une action qui, en opérant sur l'être en puissance, réalise ce qui était seulement possible et le détermine. C'est pourquoi on peut parler du mouvement aristotélicien comme coïncidence de «l'être en tant que fin » et de «la fin en tant qu'être ». Comme le remarque avec profondeur Patočka, « [1]e mouvement n'est pas dans son fond une progression de ceci vers cela, mais plutôt une manière de piétinement sur place» (Patočka 1988: 131). Cette formule d'une grande richesse phénoménologique indique que le mouvement n'est pas une sorte d' «ascension » qui actualiserait une puissance, laquelle aurait pourtant la vertu d'être « déjà là », d'exister auparavant, en tant que fin. La fin est ce vers quoi la chose s'achemine, l'existence maximale qu'un être fini peut atteindre, mais elle est également une présence qui donne force au mouvement, le telos final. Cependant, en l'accomplissant le mouvement n'en garde pas moins son caractère téléologique, potentiel, irréalisable: le telos du mouvement, le déploiement de l'être dans l'actualisation maximale de la présence dévoilée, est en même temps la force qui met en mouvement tout le processus, impulse en direction de l'apparaître un élan immanent à l'être qui appelle à sa déclôture.

Si le mouvement est une forme de piétinement sur place, il n'y a aucune raison pour qu'il s'arrête: il ne peut se poursuivre lui-même que comme mouvement. C'est parce que le mouvement va du même au même qu'il n'est pas une pure immobilité, mais l'accomplissement d'un procès, un mouvement incessant, procès infini comme manifestation ou monde. L'être n'est rien d'autre que le chemin qui mène vers luimême, l'archè est déjà un procès, le telos est procès, car, si la manifestation était un procès qui allait de ceci vers cela, le processus conduirait à son extension, le monde serait un cosmos achevé comme l'univers supra-lunaire d'Aristote. Chez Aristote lui- 
même, la continuité ontologique introduite au sein de la nature, et qui fait droit à l'être du mouvement, est en réalité compromise par la transcendance séparée du premier moteur immobile. En revanche, dans le domaine sublunaire, toute actuelle qu'elle puisse être, la fin est une potentialité «en train » de se réaliser, qui n'atteint pas vraiment son entelecheia. Ici se dessine ce que Patočka reprend sous le concept corrélatif d' "ateles energeia », acte imparfait ou incomplet. Grâce à cette relecture d'Aristote, Patočka peut substituer au modèle statique et en soi de la plénitude celui de la mobilité en tant qu'atelès energeia. Il précise en effet que «[L]'être en tant que fin est indissolublement lié au mouvement en tant qu'acte imparfait - ateles energeia » (Patočka 1988: 131), et que ce dernier caractérise le mode d'être de l'étant sensible qui n'existe que dans le processus d'actualisation de sa fin, processus qui reste pourtant approximatif. Autrement dit, l'étant est sa propre individuation et n'est pas un résultat unifié (ce qui abolirait le mouvement); l'étant est son propre «mouvement vers », il est unification, unité unifiante et non pas unité unifiée. Si la dynamis réalisée représente l'élan du mouvement, l'ateles energeia explique les limites auxquelles se confronte le mouvement lorsqu'il se déploie dans le domaine sensible, lequel est toujours en devenir. Dans la mesure où tout mouvement doit se produire dans le cadre d'un être, il se heurte à la limite imposée par l'essence de celui-ci. Alors que l'idéal du mouvement transcende toujours le cadre qui lui est imposé, il doit, dans le domaine du sensible, se restreindre à une approximation de sa fin, à une entelecheia inaccessible, une actualisation toujours incomplète. C'est cette incomplétude du mouvement, qui le fait demeurer mouvant. 


\section{REFERENCES}

ARISTOTE (1990, $1^{\text {ère }}$ éd. 1926). Physique, texte établi et trad. fr. H. Carteron, Paris : Les Belles Lettres.

ARISTOTE (1974, $1^{\text {ère }}$ éd. 1934). De Anima, trad. fr. J. Tricot, Paris : Vrin. Gallimard,

HEIDEGGER, Martin (1986). Être et Temps, trad. fr. François Vezin, Paris :

HEIDEGGER, Martin (1985). Les problèmes fondamentaux de la phénoménologie, trad. fr. J-F. Courtine, Paris : Gallimard.

HUSSERL, Edmund (1950). Idées directrices pour une phénoménologie, trad. fr. Paul Ricoeur, Paris : Gallimard.

HUSSERL, Edmund (1992). Méditations cartésiennes, trad. fr. Gabrielle Peiffer et Emmanuel Levinas, Paris : Vrin.

HUSSERL, Edmund (1976). La Crise des sciences européennes et la phénoménologie transcendantale, trad. fr. G. Granel, Paris : Gallimard.

PATOČKA, Jan (1976). Le monde naturel comme problème philosophique, trad. fr. J. Daněk et H. Declève, La Haye : Nijhoff.

PATOČKA, Jan (1991). « Martin Heidegger, penseur de l'humanité » in Epokhè $\mathrm{n}^{\mathrm{o}} 2$, Grenoble : Millon

PATOČKA, Jan (1997). Platon et l'Europe. Séminaire privé du semestre d'été 1973, trad. fr. E. Abrams, Lagrasse : Verdier.

PATOČKA, Jan (1988). Le monde naturel et le mouvement de l'existence humaine, éd. et trad. fr. E. Abrams, préface de H. Declève, Dordrecht/Boston/London: Kluwer Academic Publishers.

PATOČKA, Jan (1995). Papiers phénoménologiques, éd., intr. et trad. fr. E. Abrams, Grenoble : Million.

PATOČKA, Jan (2011). Aristote, ses devanciers, ses successeurs, trad. fr. E. Abrams, Paris : Vrin.

RODRIGO, Pierre (1995). Aristote, l'eidétique et la phénoménologie, Grenoble: Millon. 